\title{
Modeling the biomechanics of the human cornea accounting for local variations of the collagen fibril architecture
}

\author{
Andrea Montanino ${ }^{1, *}$, Alessio Gizzi ${ }^{2, * *}$, Marcello Vasta ${ }^{3, * *}$, Maurizio Angelillo ${ }^{4, \dagger}$, and Anna \\ Pandolfi ${ }^{1, \ddagger}$ \\ ${ }^{1}$ Politecnico di Milano, Department of Civil and Environmental Engineering, Piazza Leonardo da Vinci 32, Milano, Italy \\ ${ }^{2}$ University Campus Bio-Medico of Rome, Department of Engineering, Via A. del Portillo 21, Rome, Italy \\ ${ }^{3}$ University of Chieti-Pescara, Department INGEO, Viale Pindaro 42, Pescara, Italy \\ ${ }^{4}$ University of Salerno, Department of Civil Engineering, Via Giovanni Paolo II 32, Fisciano, Italy
}

Key words Human cornea, finite elements, biomechanics, hyperelasticity, distributed fibers.

\begin{abstract}
We present a finite element model of the human cornea describing the in-plane organization of the stromal collagen, modified variously to include features of the collagen architecture. We investigate numerically the implication of the local organization of collagen in the stroma on the response of the human cornea to mechanical tests. We compare four different models by simulating three ideal mechanical tests, i. e., the ex-vivo inflation test, the in-vivo probe indentation, and the in-vivo air puff tests. Numerical results show slight differences between the models in terms of global response and stress distribution. Differences in the overall mechanical response are observed in dynamic tests, while quasi-static tests are not able to differentiate between the models. Stress distributions differ markedly when a variation of the shear stiffness across the thickness is considered. We conclude that the actual architecture of the collagen across the thickness of the cornea or at the limbus has a minor relevance from the mechanical point of view with respect to the main anisotropic orthogonal collagen structure that has been considered and acknowledged in the literature.
\end{abstract}

\section{Introduction}

- Would the authors please discuss and clarify exactly which model was employed for the reference case and the variations studied? (added)

- In Fig. 7 the results are the same - indistinguishable. Indentation would produce local shearing; why does this appear to differentiate the models? (shear stress is one or two order of magnitude inferior....)

- Why is the experimentally measured curve not shown - how can we assess accuracy without it? (there is no experimental curve)

- Which model could be most representative of in vivo corneal structure. (the one with stiffness variation across the thickness)

Soft tissues biomechanics stands an active research arena for exploring novel theoretical problems including from multiscale to multiphysics challenges [11,32]. An important characteristic of soft biological media is the presence of microstructural collagen reinforcements that call for the development of advanced material models. The present study focuses on the modeling of the collagen microstructure that confers anisotropic properties to the human cornea. Advanced cornea models use constitutive equations based on well-established hyperelastic formulations and in general employ transversely isotropic material models $[9,10,25]$, either deterministic or stochastic, describing the typical structural components of soft biological tissues.

The hypothesized collagen structure of the human cornea dates back to Kokott [13] including the presence of superiorinferior (SI) and nasal-temporal (NT) preferred directions at the center of the cornea and circumferential directions at the limbus. With X-ray diffraction experiments, Meek et al. [15] first quantified the orientation of lamellae as viewed in the

\footnotetext{
* E-mail: andrea.montanino@polimi.it

** E-mail: a.gizzi@unicampus.it

*** E-mail: mvasta@unich.it

$\dagger$ E-mail: mangelil@unisa.it

¥ Corresponding author E-mail: anna.pandolfi@polimi.it, Phone: +39 022399 4217, Fax: +39 0223994200
} 
plane perpendicular to the optical axis, showing that about $49 \%$ of the stromal lamellae are preferentially aligned orthogonally along the SI and NT meridans [7]. Recent advances in biomedical imaging have revealed additional unexpected features of the collagen architecture in the human cornea [30]. Collagen lamellae in the posterior cornea are commonly twice as thick as those in the anterior [23] and interlamellar interaction results from interweaving [31]. Complex heterogeneities and distributed features of collagen fibers in the human cornea have also been documented. In [22], it has been shown that transverse shear stiffness of the anterior cornea is almost one order of magnitude greater than that the one of the posterior cornea. The measured depth-dependent transverse shear properties have been linked to decreasing degree of inclination of lamellae from the anterior to the posterior cornea. Moreover, in [1], it has been shown that the arrangement becomes less unidirectional in proximity to the anterior surface of the cornea.

In the state-of-the-art of biomechanical modeling, patient-specific numerical models including the exact collagen architecture of the human cornea are not available, though advanced models that account for the details of the corneal collagen structure have been proposed in the literature $[3,5,8,14,18,24]$. Moreover, since the accurate mechanical characterization of the material cannot be determined by simple imaging tests, an important question emerging in the definition of a predictive numerical model of the cornea concerns the ability of a particular mechanical test to reveal its micro-mechanical features. Indeed the mechanical consequences of specific collagen architectures have not been investigated deeply in the literature, especially in terms of comparison of the stress state under different possible mechanical configurations. Computational analyses often provide indications on global averaged quantities, such as a displacement component, or a displacement profile $[4,26,28]$. With a few exceptions $[19,25]$ no documentation of the stress level observed in the numerical simulations is reported; thus a comparison between different models has not be effectively done.

In the view of creating a reliable patient specific model, in this study we introduce, in an existing numerical model of the human cornea, different features of the collagen architecture that optical coherent tomography (OCT) and second harmonic generation (SHG) imaging have revealed. Thus, five different models of cornea are compared by means of numerical simulations of three ideal static and dynamic tests, i. e., the inflation test, the probe indentation test and the air puff test. For the sake of simplicity, we use the model of the cornea that we have been developing in-house, and consider a patient-specific corneal geometry acquired with a corneal topographer and already used in previous studies [27].

The paper is organized as follows. In Sec. 2 we briefly outline the cornea geometry and material model with a dedicated description of collagen architecture. Four alternative computational analyses are distinguished together with the experimental procedure simulated. In Sec. 3 we provide an extended comparison of the different models while conclusions, limitations and future perspectives are drawn in Sec. 4.

\section{Geometrical and material model}

A cornea oriented finite element code has been developed and used for numerical simulations of mechanical tests and refractive surgery interventions [18-20,25,27]. Distinguished features that characterize the software are as follows: (i) patient-specific geometries of the anterior and posterior surfaces directly derived from corneal topographies; (ii) possibility to use different material models that account for stochastic distribution of the fibrils to describe the complex architecture of reinforcing collagen in the stromal tissue; (iii) built-in identification of the stress-free configuration of the cornea; (iv) optimized customization of the main material parameters on the basis of diagnostic or postoperative measurements. The geometrical and material characteristic of the numerical model of the cornea are briefly described in the following.

\section{Geometrical model}

Patient-specific geometries of the cornea are constructed by an ad hoc software from sets of coordinates, belonging to the anterior and posterior surfaces of the cornea, supplied in digital form by an ocular topographer (Sirius, CSO, Italy) [27]. Solid geometries are automatically discretized at the desired level of refinement in standard finite elements, i. e., 8-node bricks with linear interpolation of the displacements. The following discussion refers to a particular patient-specific case selected among the ones previously used in [27], Fig. 1.

Topographer images provide the physiological (i. e., deformed by the IOP exherted by the filling gels) geometry of the cornea, which cannot be used directly in simulations. The analysis of the stress state of the cornea under IOP requires to begin with the stress-free configuration, corresponding to a null IOP. To this purpose, we adopt here an automatic procedure able to recover the stress-free configuration already described in [25]. Note that the identified stress-free geometry depends strictly upon the material properties and on the collagen architecture, thus a new identification procedure must be conducted for every change in the model.

According to the important approximations used in the previous and in the present studies, the numerical model has some limitations and potential drawbacks. First, only the thick central stroma layer is considered in the numerical calculations, whereas the thin anterior and posterior membranes, known to provide negligible contributions to the mechanical stiffness 


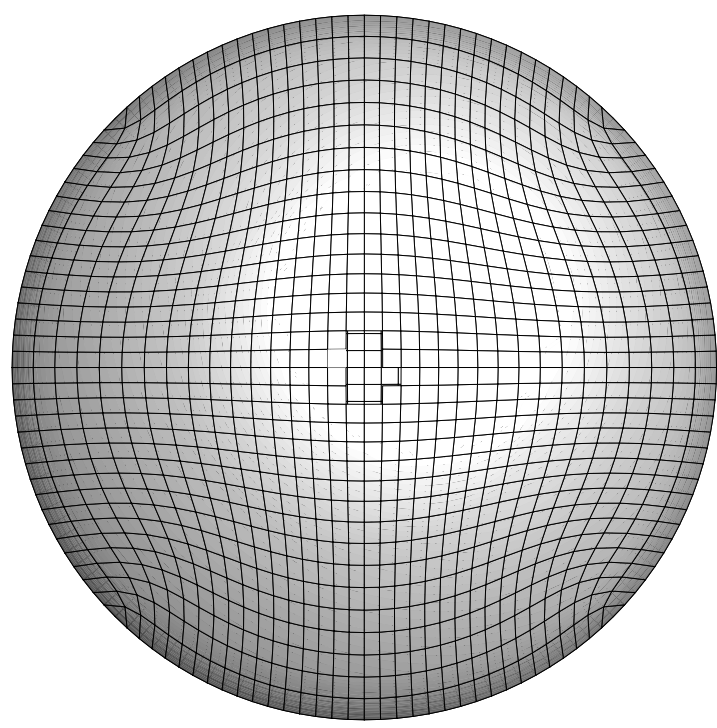

(a) Anterior view

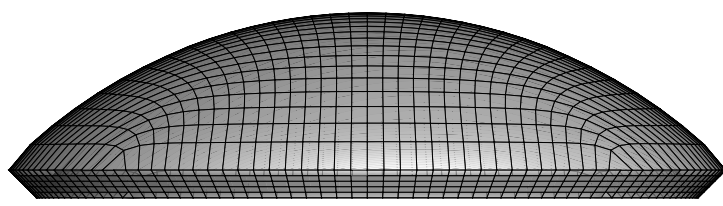

(c) Side view

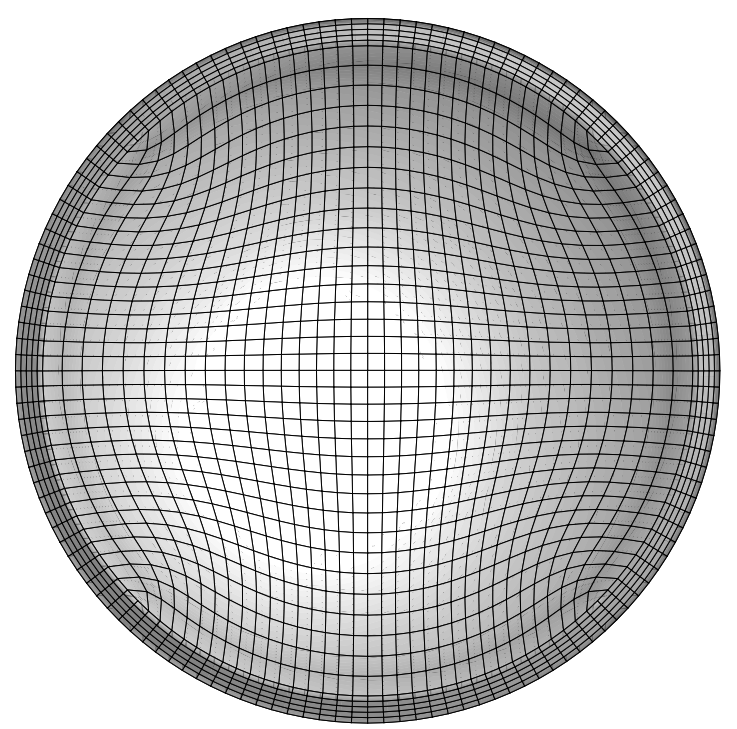

(b) Posterior view

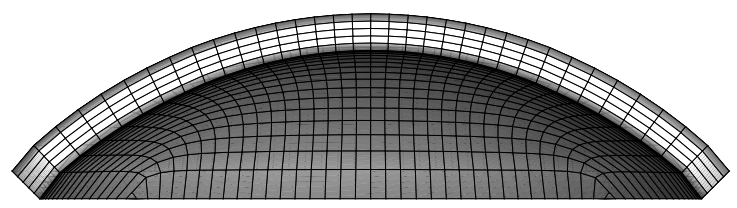

(d) Section

Fig. 1 Patient specific finite element model of the cornea. The mesh comprises 7,350 nodes and 5,780 8-node elements. The geometry refers to the stress-free configuration of a corneal topography already used in previous studies, with a finer finite element discretization [27].

of the cornea, are disregarded. Second, the geometrical model does not include the adjacent tissues, i. e., the white sclera, the iris, and the lens, which are accounted for by calibrating compliant rotational boundaries at the limbus, cf. [25]. The motivation for the exclusion of the adjacent tissues is the lack of knowledge of their in-vivo mechanical properties. Third, the model does not account for the interaction between the deformable cornea and the ocular fluids (aqueous humor) filling the anterior chamber. The motion of fluids, with the ensuing local variation of the pressure, plays an important role in fast dynamic tests, as it has been shown recently in [17]. However, for consistency with previous works and in the view of the goals of comparison between models of this study, here the presence of the fluid is accounted for by adding extra masses to the posterior nodes of the discretized cornea for all the considered models. Such masses are evaluated by considering, for each node lying on the posterior surface of the cornea, the corresponding column of fluid between cornea and iris [26,28].

\subsection{Material Model}

The material model adopted here for the stroma accounts explicitly for the presence of reinforcing collagen fibrils distributed into a matrix of proteoglycans. It is assumed that the collagen fibers are distributed according to a statistic probability density function, accounted for in the model with a second order approximation. In keeping with typical approaches adopted for biomaterials, the behaviors of the proteoglycan matrix, assumed to be quasi-incompressible, and of the reinforcing fibrils are modeled separately. Accordingly, the strain energy density function $\Psi$ is assumed to be the sum of three decoupled contributions with full separation of the arguments:

$$
\Psi=\Psi_{\mathrm{vol}}(J)+\Psi_{\text {iso }}\left(\bar{I}_{1}, \bar{I}_{2}\right)+\Psi_{\text {aniso }}\left(I_{4 M}^{*}, \sigma^{2}{ }_{M}\right) .
$$


The term $\Psi_{\text {vol }}$ depends on the jacobian $J=\operatorname{det} \mathbf{F}$, where $\mathbf{F}=\partial \mathbf{x} / \partial \mathbf{X}$ is the deformation gradient. $\Psi_{\text {vol }}$ is regarded as a penalty term to enforce weakly the incompressibility constraint and assumes the operative form

$$
\Psi_{\mathrm{vol}}(J)=\frac{1}{4} K\left(J^{2}-1-2 \log J\right),
$$

where the coefficient $K$ corresponds to a volumetric stiffness coefficient, related to the bulk modulus.

The term $\Psi_{\text {iso }}$ is intended to describe the behavior of the isotropic components of the stroma material, including the proteoglycan matrix and the portion (about 60\%) of fully dispersed fibrils, and is modelled according to Mooney-Rivlin's strain energy function

$$
\Psi_{\text {iso }}\left(\bar{I}_{1}, \bar{I}_{2}\right)=\frac{1}{2} \mu_{1}\left(\bar{I}_{1}-3\right)+\frac{1}{2} \mu_{2}\left(\bar{I}_{2}-3\right),
$$

where $\mu=\mu_{1}+\mu_{2}$ is the shear modulus of the material. The terms $\bar{I}_{1}=\operatorname{tr} \overline{\mathbf{C}}$ and $\bar{I}_{2}=1 / 2\left[(\operatorname{tr} \overline{\mathbf{C}})^{2}-\operatorname{tr}\left(\overline{\mathbf{C}}^{2}\right)\right]$ are the first and the second invariants, respectively, of the isochoric Cauchy-Green deformation tensor $\overline{\mathbf{C}}=\overline{\mathbf{F}} \overline{\mathbf{F}}^{T}$, with $\overline{\mathbf{F}}=J^{-1 / 3} \mathbf{F}$. The anisotropic term $\Psi_{\text {aniso }}$ addresses the contribution of two statistically dispersed families of collagen fibrils (representing about $40 \%$ of the total collagen), which confer an orthotropic nature to the material. The distribution of the fibril family $M$, assumed to be of von Mises type, is defined in terms of a unit vector field, $\mathbf{a}_{M}(\mathbf{x})$, identifying the main orientation of the fibrils, and of a dispersion coefficient $b_{M}(\mathbf{x})$, cf. [25]. The anisotropic strain energy function $\Psi_{\text {aniso }}$ used in the model is

$$
\Psi_{\text {aniso }}\left(I_{4 M}^{*}, \sigma^{2}{ }_{M}\right)=\sum_{M=1}^{2} \frac{k_{1 M}}{2 k_{2 M}} \exp \left[k_{2 M}\left(I_{4 M}^{*}-1\right)^{2}\right]\left(1+K_{M}^{*}\left(I_{4 M}^{*}\right) \sigma^{2}{ }_{M}\right),
$$

where $k_{1 M}$ is a stiffness parameter that controls the fibril behavior at moderate extension, and $k_{2} M$ is a dimensionless rigidity parameter that regulates the fibril behavior at large extension. The pseudo-invariants $I_{4 M}^{*}$ are defined as

$$
I_{4 M}^{*}=\mathbf{H}_{M}: \mathbf{C}, \quad \mathbf{H}_{M}=\left\langle\mathbf{A}_{M} \otimes \mathbf{A}_{M}\right\rangle=\kappa_{M} \mathbf{I}+\left(1-3 \kappa_{M}\right), \quad \mathbf{A}_{M}=\mathbf{a}_{M} \otimes \mathbf{a}_{M} .
$$

The scalar parameter $\kappa_{M}$ depends of the spatial distribution density, $\rho_{M}(\Theta)$, of the fibril orientation. According to the chosen distribution density, the material model can model fully $3 \mathrm{D}$, transversally isotropic [21], or planar, $\pi$-periodic [29], sets of fibers. In the two cases, the expression of $\kappa_{M}$ becomes, respectively,

$$
\kappa_{M}=\frac{1}{4} \int_{0}^{\pi} \rho_{M}(\Theta) \sin ^{3} \Theta d \Theta, \quad \kappa_{M}^{\mathrm{pl}}=\frac{1}{\pi} \int_{-\pi / 2}^{\pi / 2} \rho_{M}^{\mathrm{pl}}(\Theta) \sin ^{2} \Theta d \Theta .
$$

The two terms

$$
K_{M}^{*}\left(I_{4 M}^{*}\right)=k_{2 M}+2 k_{2 M}^{2}\left(I_{4 M}^{*}-1\right)^{2}, \quad \sigma^{2}{ }_{M}=\mathbf{C}:\left\langle\mathbf{A}_{M} \otimes \mathbf{A}_{M}\right\rangle: \mathbf{C}-\left(\mathbf{H}_{M}: \mathbf{C}\right)^{2},
$$

account for the variance of the fibril orientation distribution, cf. [21]. The the second order approximation of the strain energy function leads to the introduction of additional integral coefficients, which read, in the fully 3D and planar case, respectively,

$$
\widehat{\kappa}_{M}=\frac{1}{16} \int_{0}^{\pi} \rho_{M}(\Theta) \sin ^{5} \Theta d \Theta, \quad \widehat{\kappa}_{M}^{\mathrm{pl}}=\frac{1}{\pi} \int_{-\pi / 2}^{\pi / 2} \rho_{M}^{\mathrm{pl}}(\Theta) \sin ^{4} \Theta d \Theta .
$$

The hyperelastic constitutive assumptions lead to a closed-form expression of the second Piola-Kirchhoff stress tensor

$$
\mathbf{S} \equiv 2 \frac{\partial \Psi}{\partial \mathbf{C}}=\mathbf{S}_{\mathrm{vol}}+\mathbf{S}_{\mathrm{iso}}+\mathbf{S}_{\mathrm{aniso}}
$$

where the anisotropic part, related to the statistically distributed reinforcement of the structure, is given by

$$
\mathbf{S}_{\text {aniso }} \equiv 2 \frac{\partial\left\langle\Psi_{\text {aniso }}\right\rangle}{\partial \mathbf{C}}=f\left(I_{4 M}^{*}, \sigma^{2}{ }_{M}\right) \mathbf{H}_{M}+g\left(I_{4 M}^{*}, \sigma^{2}{ }_{M}\right)\left\langle\mathbf{A}_{M} \otimes \mathbf{A}_{M}\right\rangle: \mathbf{C}
$$

The coefficients of the functions $f, g$ depend upon the statistics of $I_{4}^{*}{ }_{M}$ are listed in Appendix A.

The material model used in this work is characterized by seven parameters: bulk modulus $K$, two shear moduli $\mu_{1}$ and $\mu_{2}$, two fibril stiffness $k_{1 M}$, and two fibril rigidities $k_{2} M$. Moreover, two dispersion functions $b_{M}(\mathbf{x})$ must be defined all 
Table 1 Material parameters used in the present study, cf. [27]. (pl) stands for variation on the middle cornea surface, (lb) for limbus, and (th) for variation across the thickness. $M$ stands for 1 and 2 , first and second set of fibers.

\begin{tabular}{lcccccc}
\hline Model & $K[\mathrm{MPa}]$ & $\mu_{1}[\mathrm{MPa}]$ & $\mu_{2}[\mathrm{MPa}]$ & $k_{1 M}[\mathrm{MPa}]$ & $k_{2 M}$ & $b_{M}$ \\
\hline Baseline & 5.5 & 0.08 & -0.02 & 0.05 & 200 & {$[0.2,2.8](\mathrm{pl})$} \\
ST & 5.5 & {$[0.056,0.104]$} & {$[-0.012,-0.028]$} & {$[0.0091,0.091]$} & {$[36,360]$} & {$[0.2,2.8](\mathrm{pl})$} \\
SL & 5.5 & 0.08 & -0.02 & {$[0.0091,0.091]$} & {$[36,360]$} & {$[0.2,2.8](\mathrm{pl} \& \mathrm{~b})$} \\
DT & 5.5 & 0.08 & -0.02 & 0.05 & 200 & {$[0.2,2.8](\mathrm{pl} \&$ th) } \\
ALL & 5.5 & {$[0.056,0.104]$} & {$[-0.012,-0.028]$} & {$[0.0091,0.091]$} & {$[36,360]$} & {$[0.2,2.8](\mathrm{pl} \&$ th) } \\
\hline
\end{tabular}

over the stroma domain. The set of elastic material properties calibrated for the patient group in [27] is used also in the present study, see Table 1 . The density of the stroma, needed by the dynamic analysis, is set to $\rho=1,062 \mathrm{~kg} / \mathrm{m}^{3}[28]$.

This study is focused on the collagen architecture of the stroma, in particular the goal is to quantify the mechanical differences associated to the different features recently pointed out by the literature in the field, to verify how much they are affecting the mechanical response of the cornea. The simulations illustrated here make use of a reference model (baseline) for the internal structure of the collagen distribution, describing an architecture of the fibrils which is in line with ex-vivo X-ray imaging $[2,16]$. Fibrils are strongly aligned at the center, where they follow an orthogonal organization in the NT and SI directions; at the periphery they are mostly aligned to the limbus circumference, see Fig. 2, cf. [25, 27]. In the baseline model, a fully 3D dispersion model of the fibrils is considered, with no variations across the thickness. The inplane variation of the dispersion coefficient $b_{M}(\mathbf{x})$ is visualized in Fig. 2(c), showing strong alignment in the central region and at the limbus.

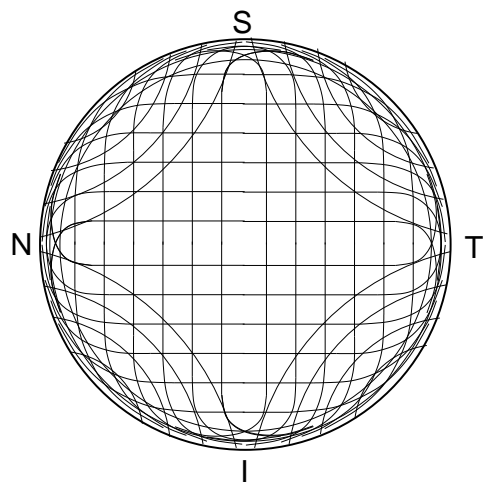

(a) Fibril structure

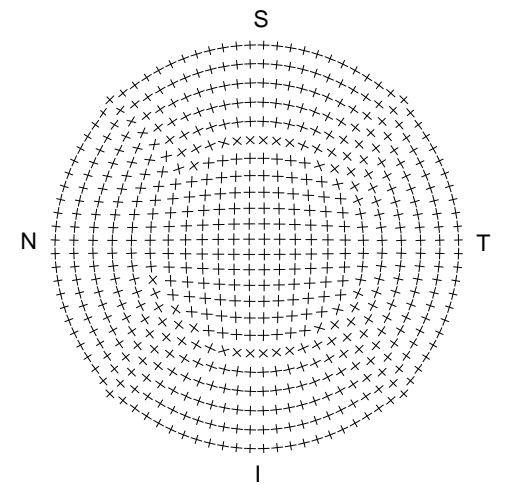

(b) Fibril main orientation

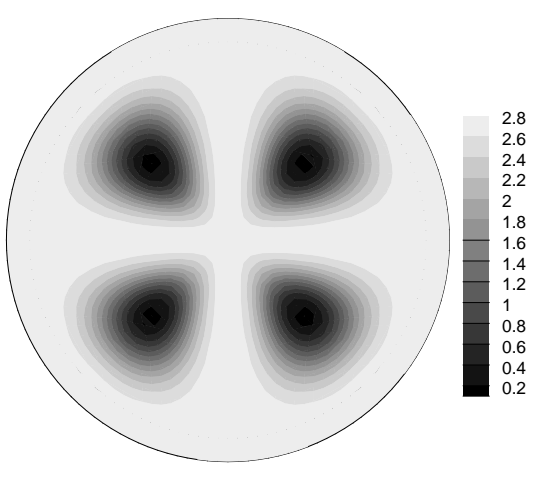

(c) Distribution parameter $b_{M}$

Fig. 2 (a) Structure of the fibril organization within the cornea, cf. [16] and [2]. S denotes the superior point, I the inferior point, $\mathrm{N}$ the nasal point and $\mathrm{T}$ the temporal point. (b) Main orientation of the fibrils assumed in the numerical model. In the central region, the two sets of fibrils have an equivalent stiffness. In the limbus region, the two sets of fibers may have a different stiffness. (c) In-plane map of the two fibril distribution parameters $b_{M}, M=1,2$.

In all the numerical models considered here, the main orientation of the fibrils described in Fig. 2(b) is preserved. Anyhow, in order to incorporate more recent findings and to evaluate their qualitative and quantitative relevance on the global mechanical response of the cornea, each model presents some difference with respect to the baseline. Specifically, we consider the following four additional models:

1. Model DT. Variation of the dispersion of the fibrils across the thickness. We use a fully 3D von Mises dispersion model, assigning different values of the dispersion coefficient varying linearly from the anterior $\left(b_{M \text { min }}=0.2\right)$ to the posterior $\left(b_{M \max }=2.8\right)$ surface, as a simplified trial to reflect the indication reported in [6]. In particular, the map of Fig. 2(c) applies only to the posterior surface, while in the anterior surface is assumed a constant coefficient $b_{M}=0.2$.

2. Model SL. Variation of the stiffness and of the degree of dispersion of the fibrils at limbus. The dispersion coefficient of the fibrils at limbus (i. e., fibers belonging to the two external rings of elements in Fig. 1) is varied from $b=0.2$ in the anterior surface to $b=2.8$ in the posterior surface, thus the aligned fibers at limbus are only considered in the 
posterior side. Moreover, the stiffness parameters of the fibrils running circumferentially in the deepest one third of the limbus thickness are set 10 times larger than the stiffness parameters of the fibrils oriented radially, to account for the observation reported in [1].

3. Model ST. Variation of the stiffness across the thickness. Experimental observation [22] measured a transverse shear modulus of the stroma ten times higher in the anterior third of the cornea than in the posterior third, due to the different level of interweaving of the lamellae. The stiffness parameters of the two terms that account for the presence of the fibrils, i. e., $\Psi_{\text {iso }}$ (for the $60 \%$ uniformly dispersed) and $\Psi_{\text {aniso }}$ (for the $40 \%$ more aligned), are assumed to vary linearly across the thickness, as so as the stiffness contribution due to collagen in anterior stroma is 10 times larger than the one in the posterior stroma.

4. Model ALL. It accounts for the three modifications in a whole.

The parameters of the five models used in the numerical analyses are listed in Table 1. In the case of non constant values, Fig. 2(c) shows the in-plane range of variability; the variability across the thickness is linear.

\section{Static and Dynamic analysis}

Since the goal of the cornea models is their use in supporting clinical applications, the comparison between the five different models is achieved through the simulation of two in-vivo mechanical contact and contactless tests. Mechanical tests induce important deformations localized at the center of the cornea, with no damage for the tissues. As initial comparative verification of the five models, an ideal in-vitro inflation test has been simulated, where the cornea is loaded from the posterior with a IOP growing hypothetically from 0 to $40 \mathrm{mmHg}$.

Before conducting the simulations, the stress-free configuration of each cornea model has been identified through the iterative procedure described in [25]. The stress-free configuration is fundamental to assess qualitatively the stress and strain distribution; the identified unstressed geometry has been used subsequently for the simulation of quasi-static and dynamic tests.

Simulations of in-vivo tests require modeling from the physiological configuration. Thus each simulation begins with an initial quasi-static analysis where the cornea is progressively pressurized up to the physiological IOP, set in these calculation to $16 \mathrm{mmHg}=2.13 \mathrm{kPa}$. The boundary conditions imposed to the nodes at the limbus allow the free rotation of the cornea cross section about the limbus, optimizing the difference between the current model and a model that includes limbus and sclera, see [19]. The simulation of the in-vivo test begins once the physiological state has been reached.

For the static test, the action of an opto-mechanical testing device applied at the corneal apex is modeled in terms of a displacement history imposed to the nodes touched by the mechanical probe. The probe is a $0.5-\mathrm{mm}$ diameter cylindrical indenter with a hemispherical tip [22]. The loading procedure consists in advancing the mechanical probe up to $600 \mu \mathrm{m}$ into the cornea. The process is subdivided in 60 steps. Static analysis are conducted using an explicit solver.

For the dynamic test, the action of a contactless ocular tonometer (CorVis ST), that uses a localized air jet to cause the oscillation of the cornea, is modelled by applying an estimated pressure history on a limited area of the central anterior cornea. The sudden pulse exerted by the instrument causes the inward motion of the cornea, which passes through an applanation, and successively snaps into a slight concavity. When the air pulse pressure decreases, the elastic corneal tissue recovers the original configuration, passing through a second applanation. Although the actual space and time profile of the air jet pressure and its maximum value are not provided by the instrument, the imprint of the air jet on the anterior corneal surface has been estimated, through preliminary parametric analyses, using analytical expressions [28]. The air jet pressure is applied over a $1.5 \mathrm{~mm}$ radius (or $3.0 \mathrm{~mm}$ diameter) circular area centered at the apex of the cornea. The pressure has the functional form

$$
p(t, r)=p_{0} \exp \left[-64\left(\frac{t}{T}-\frac{1}{4}\right)^{2}\right] \exp \left(-0.44 r^{2}\right),
$$

where $p_{0}=40 \mathrm{kPa}$ is the maximum air jet pressure, $T / 2=20 \mathrm{~ms}$ the duration of the air jet, and $r$ the distance in mm from the center of the jet of a point on the corneal surface, cf. [28]. Note that the dynamical test is not correctly modelled, since the fluids filling the anterior chamber of the eye have been disregarded for the sake of simplicity. Thus the effects of fluid-structure interaction are not accounted for and the numerical analysis is not able to capture the dynamics of the final part of the test, where the inertia of the fluid causes a delay in the motion of the cornea. This issue is currently tackled in a parallel work [17]. The time integration of the dynamical analysis is achieved with a central difference time stepping algorithm. 


\section{Results}

\subsection{Inflation test}

Figure 3 shows the results of the inflation test for the five models, in terms of IOP versus the displacement of the cornea apex. All curves superpose well to the reference model up to the physiological IOP $(16 \mathrm{mmHg})$, revealing a maximum relative displacement less than $2 \mu \mathrm{m}$. However, at higher IOP the five curves show marked differences, and are characterized by a stiffer response (ST model) or by a more compliant response (DT model) with respect to the baseline model, see Fig. 3. The similarity of the response at the physiological IOP is highlighted in Fig. 4, where the numerically computed corneal profiles along the NT and SI meridian are compared at the physiological IOP and at the maximum inflation pressure. Differences between the geometrical configurations associated to the five models cannot be appreciated, except for the ST and DT models at $40 \mathrm{mmHg}$. More significantly, Fig. 5 compares the normal stress distribution for the first four models (ALL provides the same figure as ST and is not reported). Interestingly, while there is no evident stress difference between the first three models, the SL and ALL models show a marked variation of the stress across the thickness and, specifically, a reverse trend: the maximum tensile stress is found on the anterior side of the cornea, as expected from a heterogeneous, layered shell with higher stiffness in the anterior side. Note that here and in the following tests, the stress distribution across the thickness has been reported only for the NT meridian. In general, no stress difference is observed along the SI meridian, although the geometry of the cornea considered here is not axis-symmetric.

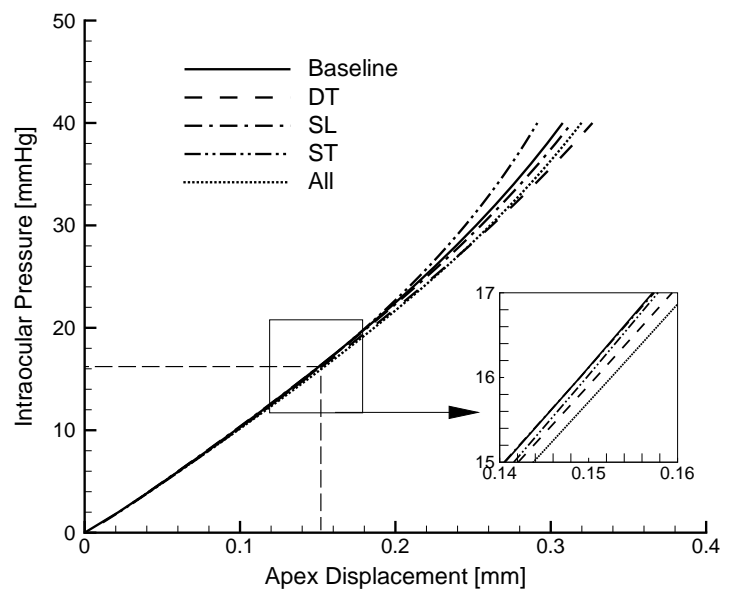

Fig. 3 Inflation test. Intraocular pressure versus apex displacement. The thick line represents the response of the baseline model.

The results of the simulations of quasi-static contact tests are shown in Figs. 6-8. Fig. 6 compares the global mechanical response of the five models in terms of probe force versus probe displacement, which correspond to the displacement of the corneal apex. In the whole range of the imposed displacements, $0.6 \mathrm{~mm}$, the curves corresponding to the SL, ST and ALL models show a more compliant behavior than the baseline model, while the DT model replicates the baseline model behavior. The small differences in the mechanical response to the probe action can be appreciated in Fig. 7, where the corneal profiles along the NT and SI meridian obtained for the five models are visualized. Note that the probe test obliges the cornea to reach a particular shape and position in the central part, following the shape of the probe. This hinders the possibility to observe marked differences in the profiles of the five models. However, a more compliant behavior is vaguely observed for the ST and ALL models. The higher compliance of ST and ALL models is due certainly to the fact that the structure is loaded in a non-natural way. The existence of a gradient in the stiffness across the thickness results in a lower bending performance of the shell. Fig. 8 compares the normal stress distribution of the five models in correspondence to the maximum probe displacement. Models baseline, DT and SL predict a stress reversal with tensile stress at the posterior side of the cornea and compressive stress at the probe contact point. ST and ALL models, instead, show a less marched variation of the stress across the thickness, due to the gradient of stiffness. Interestingly, during the probe stress the shear stress components remain one or two orders of magnitude smaller than the normal stresses. 


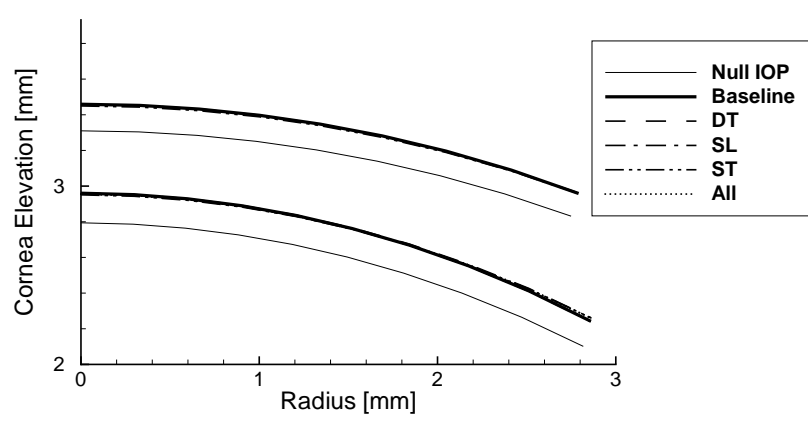

(a) NT: $16 \mathrm{mmHg}$

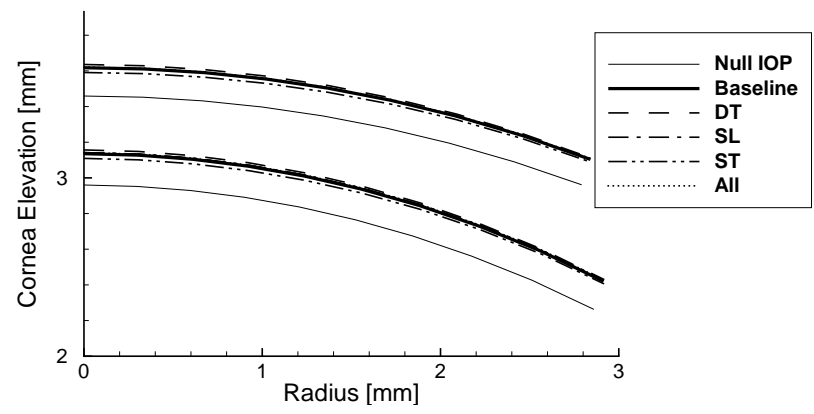

(c) NT: $40 \mathrm{mmHg}$

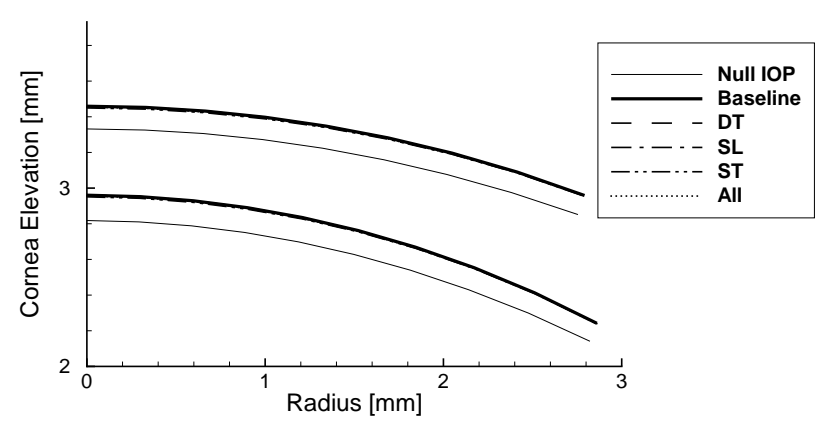

(b) SI: $16 \mathrm{mmHg}$

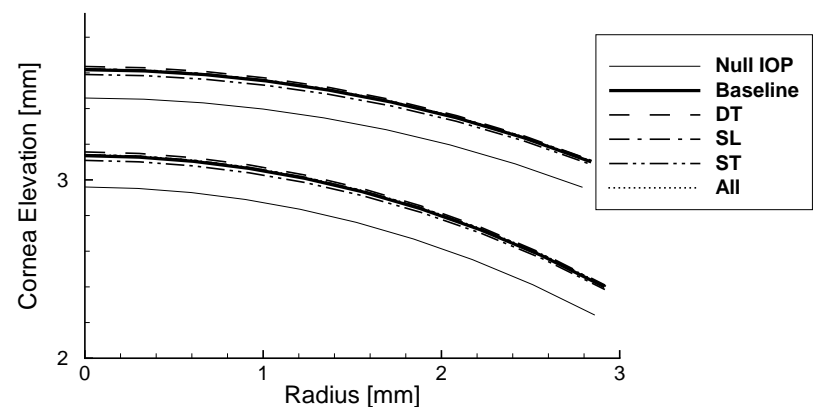

(d) SI: $40 \mathrm{mmHg}$

Fig. 4 Inflation test. Comparison of the anterior and posterior profiles of the cornea along the (a,c) Nasal-Temporal meridian (from the center to Temporal side); (b,d) Superior-Inferior meridian (from the center to the superior side). (a,b) Physiological IOP. Thin lines visualize the posterior and anterior surfaces of the baseline model of the cornea at zero IOP. Because of the unstressed configuration identification procedure, all curves superpose at the physiological IOP. (c,d) Maximum inflation. Thin lines visualize the anterior and posterior surface of the cornea at the physiological IOP.
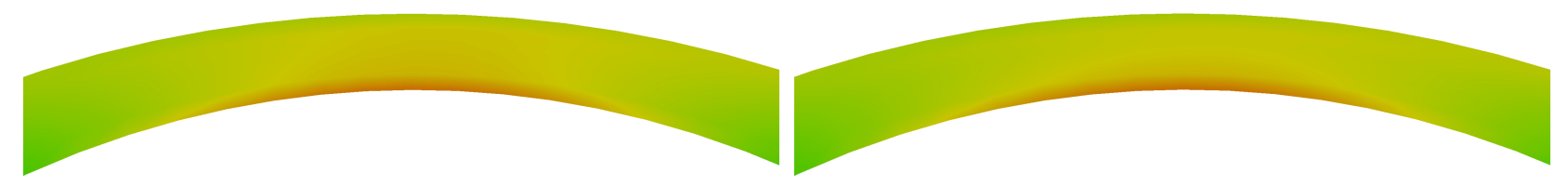

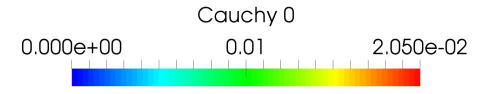

(a) Baseline

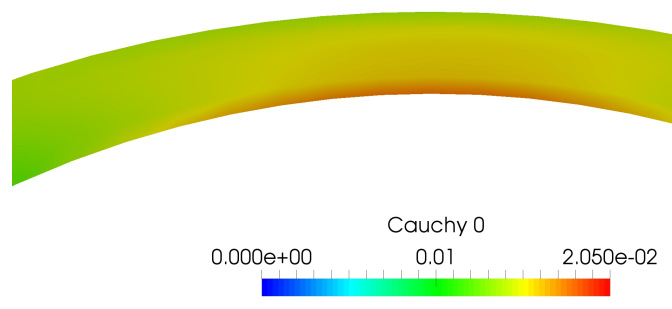

(c) $\mathrm{SL}$

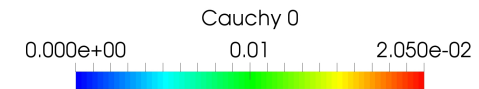

(b) DT
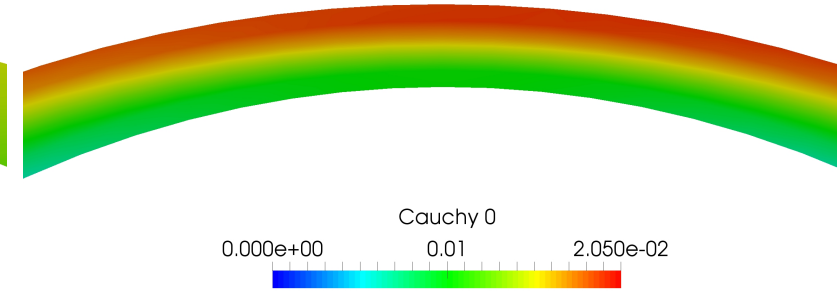

(d) ST and ALL

Fig. 5 Inflation test. Comparison of the NT stress distribution at the physiological IOP over the NT meridian section for the five models. The only different behavior is observed in the ST and ALL models where the stiffer anterior layer carries almost all the tensile stress, leading to a reverse variation (maximum tensile stress on the anterior side). The ST and ALL models behaves essentially in the same way, since both include specific stiffness variability across the thickness. The stress distribution along the SI meridian for the five models shows the same trend. 


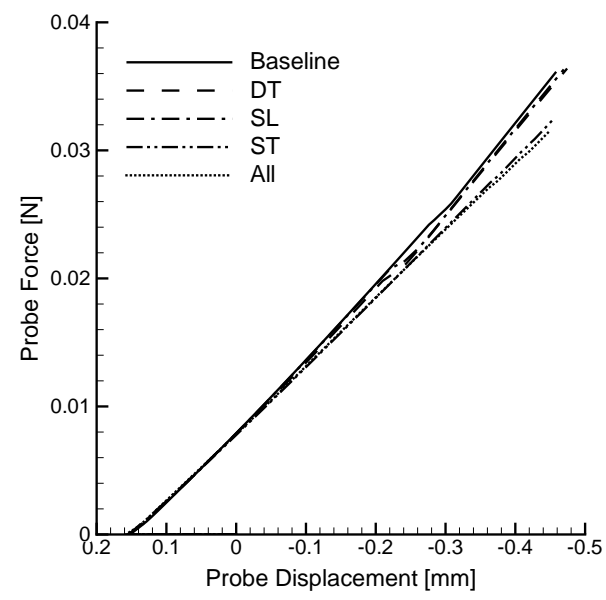

Fig. 6 Probe test. Force versus apex displacement curves. ALL models show a more compliant behavior with respect to the baseline model, except for the DT model that exactly replicates the baseline model behavior.

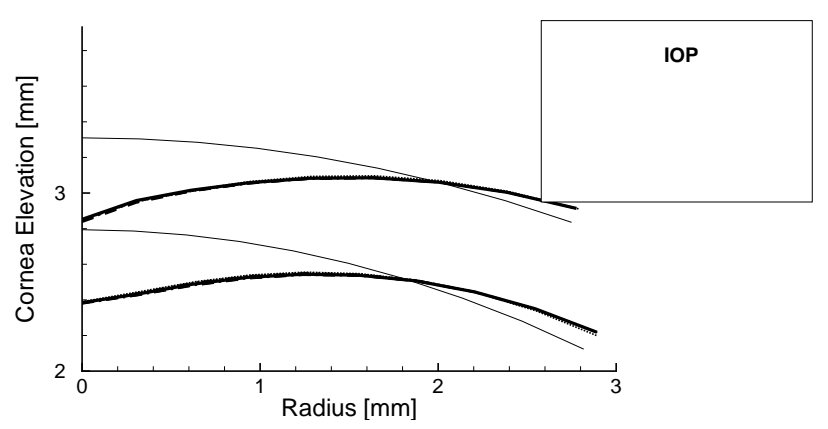

(a) NT

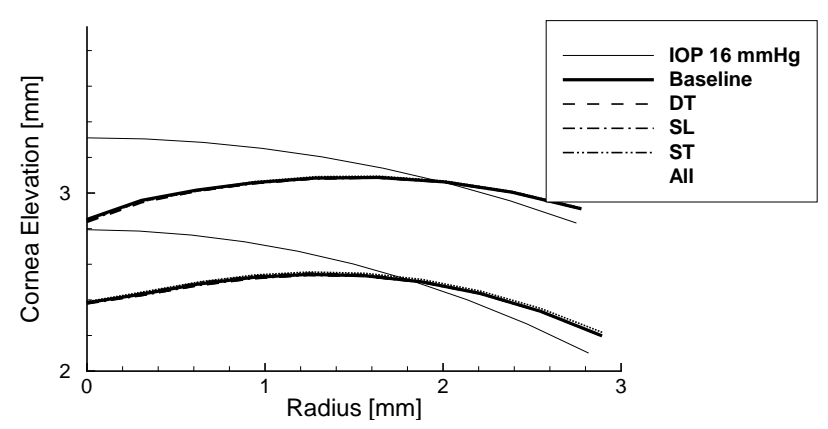

(b) SI

Fig. 7 Probe test. Cornea profiles at the maximum displacement of the probe, corresponding to $0.6 \mathrm{~mm}$ indentation of the cornea, along the NT and the SI meridian, respectively. Two thin lines refer to the anterior and posterior surfaces of the cornea at the physiological IOP $=16 \mathrm{mmHg}$.

\subsection{Dynamic contactless test}

The results of the simulations of dynamic contactless tests are visualized in Figs. 9-11. Fig. 9 compares the response of the five models considering the displacement of the cornea apex. Fig. 9(a) shows the time history of the apex displacement for the five models. Fig. 9(b) shows the mechanical response in terms of air jet pressure versus apex displacement. In contrast with the quasi-static tests, dynamical tests highlight a different response between the five models. This is particularly evident in Fig. 10 where the five configurations in correspondence to the maximum value of the air jet pressure are compared. Note that all the five models show a more compliant dynamical behavior with respect to the baseline model. Interestingly, the model that accounts for all the variants in the collagen structure behaves clearly as the most compliant, showing a larger displacement at all times, while it is hard to differentiate among the DT, SL and ST models.

\section{Conclusions}

By considering a particular hyperelastic multi-component material model that includes the stochastic description of the dispersion of the collagen fibrils, the present study has focused on the comparison of the mechanical response of five models of the human cornea, differing variously for the collagen stiffness, organization and dispersion across the thickness and at the limbus. Aim of the study was to ascertain the ability of a mechanical test to distinguish between different collagen architectures, in the view of the definition of an identification procedure that includes not only the material constants but also the underlying microstructure of the cornea. 

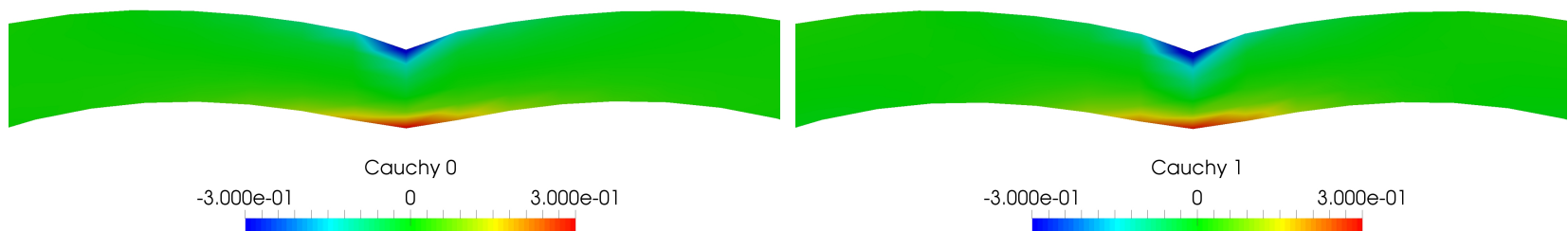

(a) Baseline

(b) DT

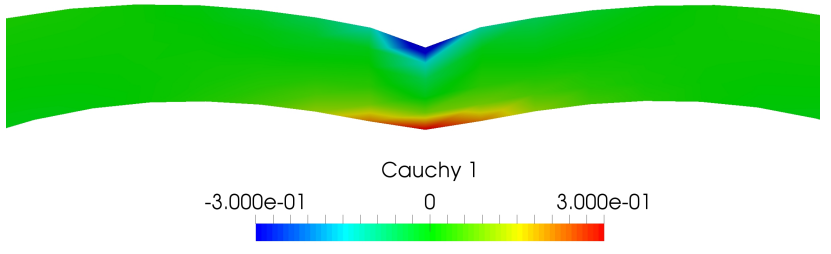

(c) SL

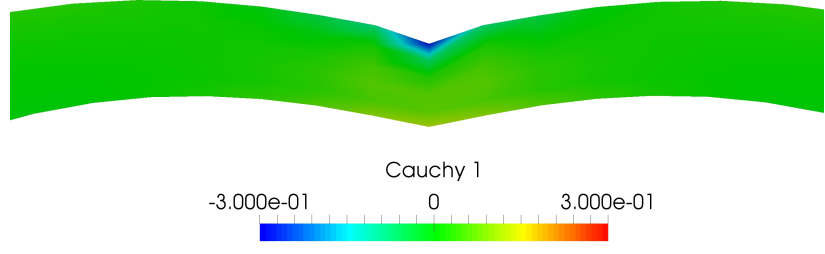

(d) ST and ALL

Fig. 8 Probe test. Comparison of the stress distribution at the maximum indentation along the NT meridian section for the five models. Also in this test, the only different behavior is observed in the ST and ALL models, where the variability of the stroma stiffness across the thickness provides a reduction of the maximum stresses both in the tensile and compressive side.

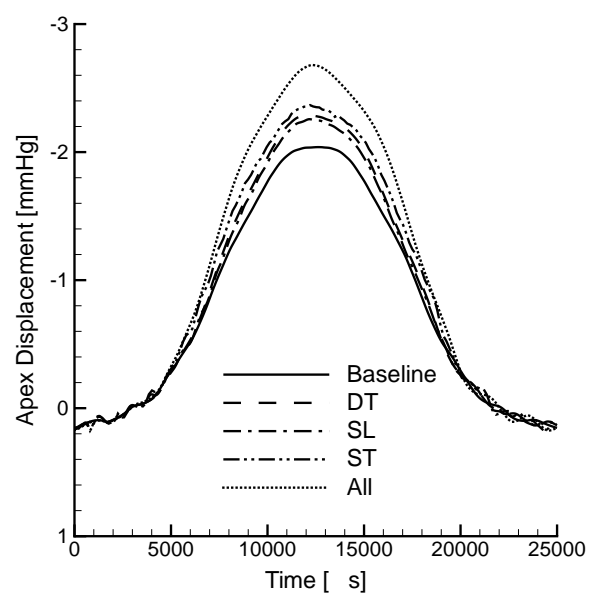

(a)

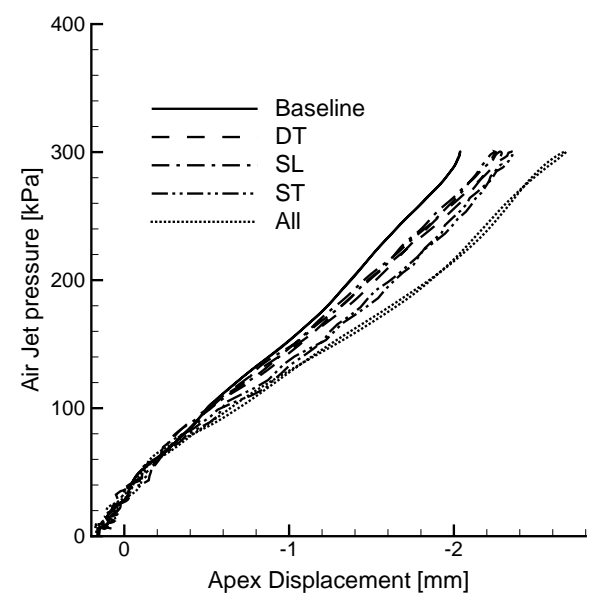

(b)

Fig. 9 Dynamic contactless test for the five models. (a) Cornea apex displacement time history. (b) Air jet pressure versus apex displacement curve.

A baseline model, adopted in recent studies [25,27], has been taken as reference. The patient-specific geometry obtained from a corneal topographer has been transformed into a solid model of the physiological configuration of the cornea and then discretized into finite element with a reasonably fine mesh size.

Four variants of the baseline model account for different levels of dispersion of the collagen across the thickness (DT model), for the variability of the stiffness of the stroma in proximity of the limbus (SL model) and across the thickness (ST model), or for the three features simultaneously (ALL model). The five models have been compared by means of numerical simulations of quasi-static and dynamic tests.

Numerical results showed that the inflation test does not allow to highlight differences between the models, except for the models where the stiffness of the material has been varied across the thickness (models ST and ALL). The mechanical 


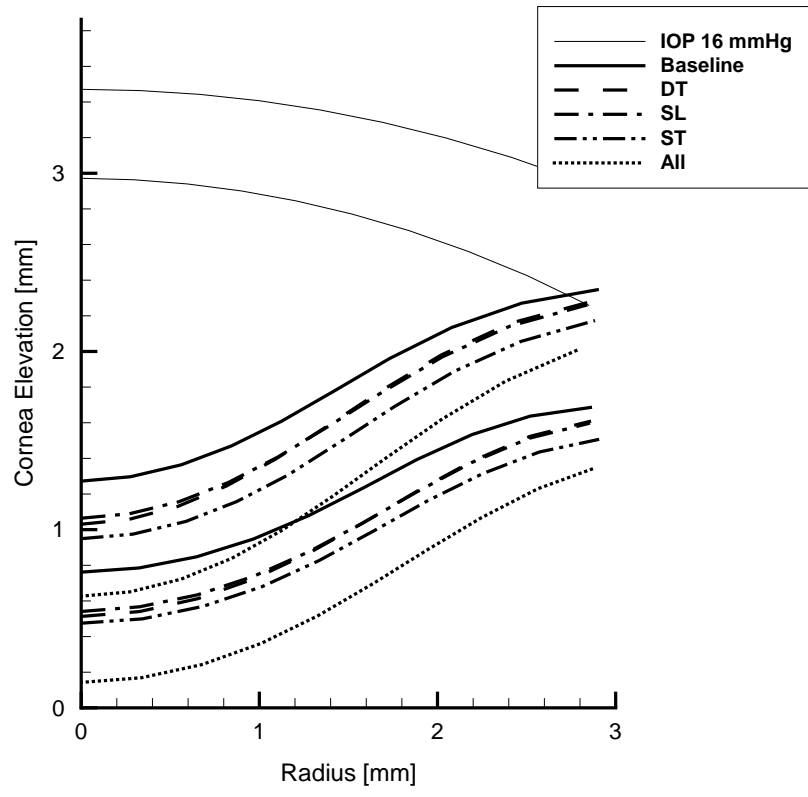

(a) NT

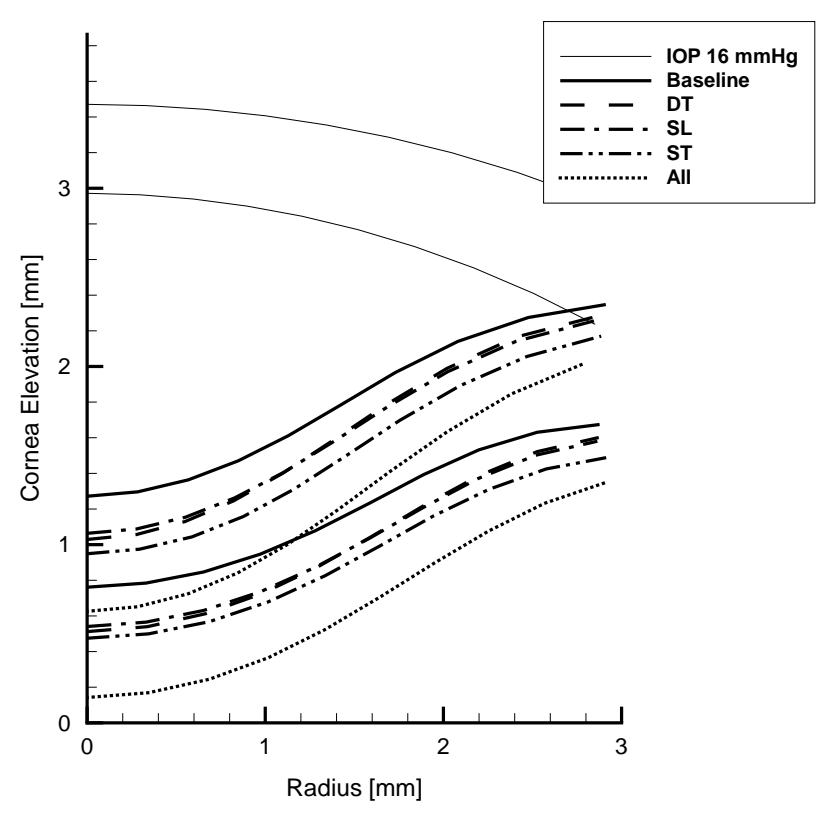

(b) SI

Fig. 10 Dynamic contactless test. Comparison of the cornea profiles at the maximum value of the air jet for the five models. Two thin lines visualize the anterior and posterior surfaces of the cornea at the physiological IOP.

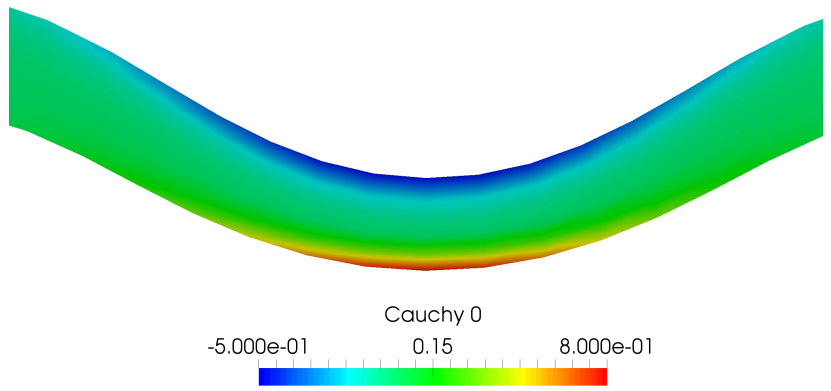

(a) Baseline

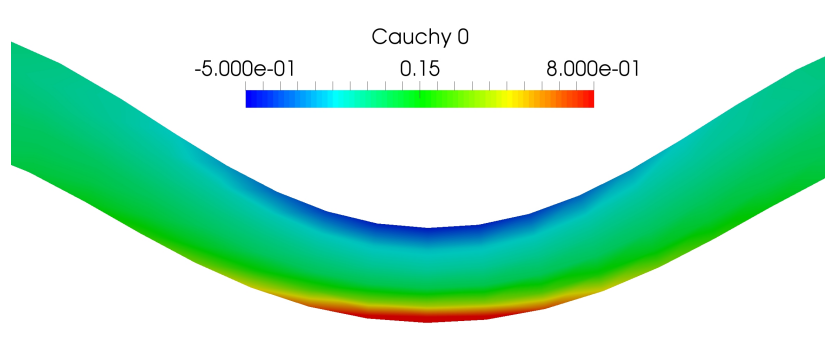

(c) SL

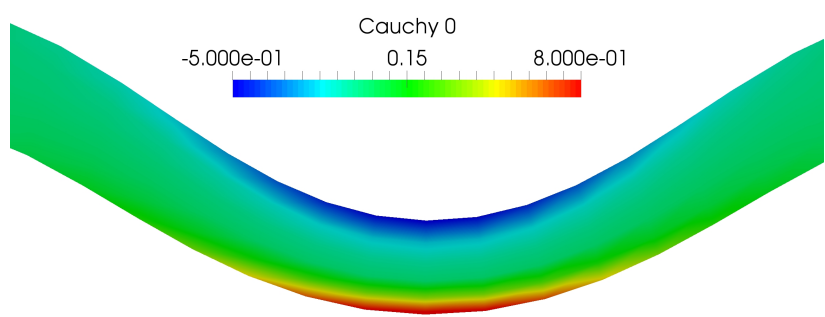

(b) $\mathrm{DT}$

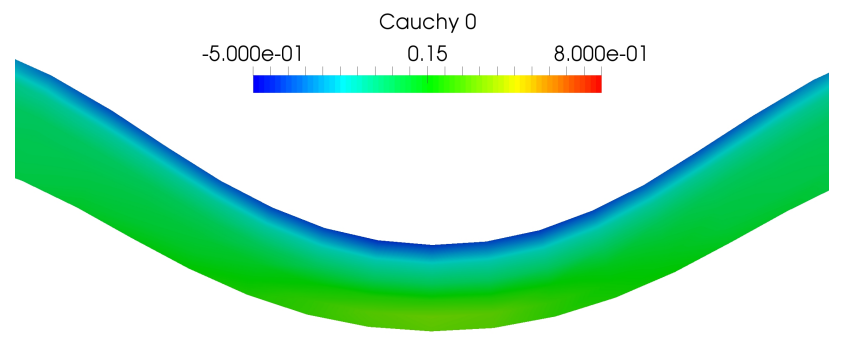

(d) ST and ALL

Fig. 11 Dynamic contactless test. Comparison of the NT stress distribution at the physiological IOP over the NT meridian section for the five models. A different behavior is observed in SL and ALL models, where the variable stiffness helps in reducing the stress gradient across the thickness. 
response differentiates only at high, non-physiological, pressures, Figs. 4(c)-4(d), but realistically in-vitro tests cannot be used in clinical applications for the characterization of material properties.

The probe test is able to point out only slight differences between the models. Numerical results exclude appreciable differences between the mechanical responses of baseline and DT models; while ST and ALL models maniest a larger compliance, cf. Figs. 7(a)-7(b). Finally, the localized pressure of the air puff test clarified that ST and ALL models are characterized by a higher dynamical compliance, while the baseline model the highest dynamical stiffness.

According to the results of the present study, the variability of the collagen dispersion across the thickness and the collagen organization at the limbus are not of primary relevance in the static and dynamic mechanical response of the corneal shell. In fact, among the four alternative models, the models DT and SL, that include a variability of the dispersion across the thickness and at limbus, show a behavior very close to the one of the baseline model. Contrariwise, the main mechanical features of the cornea derive from the variability of the stiffness of the material across the thickness, cf. the results of the models ST and ALL.

Numerical results highlight that the stress distribution changes exclusively when the material stiffness is modified, while no appreciable differences with respect to the baseline mode can be observed when the fiber dispersion is altered [22]. This suggests that the presence of a gradient of the material stiffness across the thickness is the most important feature among the ones here considered. Moreover, it can be supported by a mechanical justification. Intuitively, a stiffer anterior layer helps in enhancing the mechanical protective function of the cornea against unusual loads, e.g., the impact with a flying object or a trauma. The beneficial effect of a stiffer external layer is observed in the simulation of static and dynamic tests where the natural configuration of the cornea is reversed: the magnitude of the stresses is reduced overall.

We conclude by pointing out the important drawback of the models, that actually affects only the dynamical tests: the filling fluids, which indeed play an important role in the mechanical response of the system, are not considered in a proper way, but only as added masses that increase the inertia of the cornea during the motion. To tackle this issue, a coupled fluid-structure interaction approach, based on meshfree discretization of the fluid domain, has been developed in 2D [17] and the $3 \mathrm{D}$ version is currently under development.

\section{References}

[1] M. Abahussin and et al., 3D collagen orientation study of the human cornea using x-ray diffraction and femtosecond laser technology, Invest. Ophthalmology. Vis. Sci. 50, 5159-5164 (2009).

[2] H. Aghamohammadzadeh, R. H. Newton, and K. M. Meek, X-ray scattering used to map the preferred collagen orientation in the human cornea and limbus, Structure 12(2), 249-256 (2004).

[3] V. Alastrué, B. Calvo, E. Pena, and M. Doblaré, Biomechanical modeling of refractive corneal surgery, J. Biomech. Eng. 128, 150-160 (2006).

[4] M. A. Ariza-Gracia, J. F. Zurita, D. P. Pinero, J. F. Rodriques-Matas, and B. Calvo, Coupled biomechanical response of the cornea assessed by non-contact tonometry. A simulation study, PLoS ONE 10, e0121486 (2015).

[5] D. Cabrera Fernández, A. M. Niazi, R. M. Kurtz, J. P. Djotyan, and T. Juhasz, Finiteelement analysis applied to cornea reshaping, J. Biomed. Optics. 10, 064018 (2005)

[6] X. Cheng and P. M. Pinsky, Mechanisms of self-organization for the collagen fibril lattice in the human cornea, J. R. Soc. Interface 10, 20130512 (2013).

[7] A. Daxer and P. Fratzl, Collagen fibril orientation in the human corneal stroma and its implication in keratoconus, Invest. Ophthalmology. Vis. Sci. 38, 121-129 (1997)

[8] A. Elsheikh, Finite element modeling of corneal biomechanical behavior, J. Refract. Surg. 26(4), 289-300 (2010).

[9] S. Federico, Porous materials with statistically oriented reinforcing fibres, Nonlinear Mechanics of Soft Fibrous Materials 559, 49-120 (2015).

[10] S. Federico, A. Grillo, G. La Rosa, G. Giaquinta, and W. Herzog, A transversely isotropic, transversely homogeneous microstructural-statistical model of articular cartilage, J. Biomech. 38, 2008-2018 (2005).

[11] S. Federico and W. Herzog, Towards an analytical model of soft tissues, J. Biomech. 41, 3309-3313 (2008).

[12] A. Gizzi, A. Pandolfi, and M. Vasta, Statistical characterization of the anisotropic strain energy in soft materials with distributed fibers, Mec. Mat. 92, 119-138 (2016).

[13] W. Kokott, Über mechanisch-funktionelle Strukturen des Auges, Albrecht von Graves Archiv für Ophthalmologie 138(4), 424485 (1938)

[14] E. Lanchares, B. Calvo, J. Cristóbal, and M. Doblaré, Finite element simulation of arcuate for astigmatism correction, J. Biomech. 41(4), 797-805 (2008)

[15] K. M. Meek, T. Blamires, G. F. Elliot, T. J. Gyi, and C. Nave, The organisation of collagen fibrils in the human corneal stroma: a synchrotron X-ray diffraction study, Curr. Eye Res. 6, 841-846 (1987).

[16] K. M. Meek and C. Boote, The use of X-ray scattering techniques to quantify the orientation and distribution of collagen in the corneal stroma, Prog. Retin. Eye Res. 28(5), 369-392 (2009).

[17] A. Montanino, M. Angelillo, and A. Pandolfi, Modeling with a meshfree approach the cornea-aqueous humor interaction during the air puff test, J. Mech. Behav. Biomed. Mater. https://doi.org/10.1016/j.jmbbm.2017.05.042, 1-23 (2017). 
[18] A. Pandolfi, G. Fotia, and F. Manganiello, Finite element simulations of laser refractive corneal surgery, Eng. Comput. 25, 15-24 (2009).

[19] A. Pandolfi and G. A. Holzapfel, Three-dimensional modeling and computational analysis of the human cornea considering distributed collagen fibril orientations, J. Biomech. Eng. 130, 061006 (2008).

[20] A. Pandolfi and F. Manganiello, A model for the human cornea: constitutive formulation and numerical analysis, Biomech. Model. Mechanobiol. 5, 237-246 (2006).

[21] A. Pandolfi and M. Vasta, Fiber distributed hyperelastic modeling of biological tissues, Mec. Mat. 44, 151-162 (2012).

[22] S. J. Petsche, D. Chernyak, J. Martiz, M.E. Levenston, and P. M. Pinsky, Depth-dependent transverse shear properties of the human corneal stroma, Invest. Ophthalmology. Vis. Sci. 53, 873-880 (2012).

[23] A. J. Quantock and et al., Small-angle fibre diffraction studies of corneal matrix structure: A depth-profiled investigation of the human eye-bank cornea, J. Appl. Crystallography. 40, s335-s340 (2007).

[24] A. S. Roy and W. J. J. Dupps, Patient-specific modeling of corneal refractive surgery outcomes and inverse estimation of elastic property changes, J. Biomech. Eng. 133, 011002 (2011).

[25] P. Sánchez, K. Moutsouris, and A. Pandolfi, Biomechanical and optical behavior of human corneas before and after photorefractive keratectomy, J. Cataract. Refract. Surg. 40, 905-917 (2014).

[26] I. Simonini, M. Angelillo, and A. Pandolfi, Theoretical and numerical analysis of the corneal air puff test, J. Mech. Phys. Sol. 93, 118-134 (2016).

[27] I. Simonini and A. Pandolfi, Customized finite element modelling of the human cornea, PloS ONE 10, e0130426 (2015).

[28] I. Simonini and A. Pandolfi, The influence of intraocular pressure and air jet pressure on corneal contactless tonometry tests, J. Mech. Behav. Biomed. Mater. 58, 75-89 (2016).

[29] M. Vasta, A. Gizzi, and A. Pandolfi, On three- and two-dimensional fiber distributed models of biological tissues, Prob. Eng. Mrch. 37, 170-179 (2014).

[30] M. Winkler and et al., Nonlinear optical macroscopic assessment of 3-D corneal collagen organization and axial biomechanics, Invest. Ophthalmology. Vis. Sci. 52, 8818-8827 (2011).

[31] G. Wollensak, E. Spörl, C. Mazzotta, T. Kalinski, and S. Sel, Interlamellar cohesion after corneal crosslinking using riboflavin and ultraviolet A light, British. J. Ophthalmology. 95, 876-880 (2011).

[32] J.Z. Wu, W. Herzog, and S. Federico, Finite element modeling of finite deformable, biphasic biological tissues with transversely isotropic statistically distributed fibers: toward a practical solution, Zeitschrift für angewandte Mathematik und Physik 67, 26 (2016).

\section{A Appendix}

The coefficients entering the anisotropic second Piola-Kirchhoff stress tensor in (10) are:

$$
f\left(I_{4 M}^{*}, \sigma^{2}{ }_{M}\right)=\Psi_{\text {aniso }}^{*} \sum_{j}^{0.3} a_{j} I_{4 M}^{* j}, \quad g\left(I_{4 M}^{*}, \sigma^{2}{ }_{M}\right)=\Psi_{\text {aniso }}^{*} \sum_{j}^{0 . .2} b_{j} I_{4 M}^{* j}
$$

329 with $k_{2 M} \equiv k_{2}$ and $\sigma^{2}{ }_{M} \equiv \sigma^{2}$ for the sake of notation

$$
\begin{aligned}
& a_{0}=-4 k_{2}-12 \sigma^{2} k_{2}^{2}-8 \sigma^{2} k_{2}^{3}, \\
& a_{1}=-8 k_{2}^{2}+12 \sigma^{2} k_{2}^{2}+24 \sigma^{2} k_{2}^{3}, \\
& a_{2}=16 k_{2}^{2}-24 \sigma^{2} k_{2}^{3}, \\
& a_{3}=-8 k_{2}^{2}+8 \sigma^{2} k_{2}^{3}, \\
& b_{0}=4 k_{2}+8 k_{2}^{2}, \\
& b_{1}=-16 k_{2}^{2}, \\
& b_{2}=8 k_{2}^{2},
\end{aligned}
$$

For details about the derivation we refer to the original works $[12,21,29]$ where also the corresponding tangent stiffness is provided. 